\title{
OPTIMIZATION OF DOPANT DIFFUSION AND ION IMPLANTATION TO INCREASE INTEGRATION RATE OF FIELD-EFFECT HETEROTRANSISTORS. AN AP- PROACH TO SIMPLIFY CONSTRUCTION OF THE HET- EROTRANSISTORS
}

\author{
E.L. Pankratov ${ }^{1,3}$, E.A. Bulaeva ${ }^{1,2}$ \\ ${ }^{1}$ Nizhny Novgorod State University, 23 Gagarin avenue, Nizhny Novgorod, 603950, \\ Russia \\ ${ }^{2}$ Nizhny Novgorod State University of Architecture and Civil Engineering, 65 Il'insky \\ street, Nizhny Novgorod, 603950, Russia \\ ${ }^{3}$ Nizhny Novgorod Academy of the Ministry of Internal Affairs of Russia, 3 Ankudi- \\ novskoe Shosse, Nizhny Novgorod, 603950, Russia
}

\begin{abstract}
In this work we introduce an approach to decrease dimensions of a field-effect heterotransistors. The approach based on manufacturing field-effect transistors in heterostructures and optimization of technological processes. At the same time we consider possibility to simplify their constructions.
\end{abstract}

\section{KEYWORDS}

Field-effect heterotransistors; simplification of construction of heterotransistors; increasing integration rate of transistors; optimization of manufacturing of heterotransistors; analytical modelling of technological process

\section{INTRODUCTION}

One of the actual questions of the solid state electronics is increasing of integration rate of elements of integrated circuits [1-14]. At the same time with decreasing of integration rate of elements of integrated circuits one can find decreasing of dimensions of the elements. In the present time it is known several approaches to decrease dimensions of elements of integrated circuits and their discrete analogs. Two of them are laser and microwave types of annealing of dopants and radiation defects [15-17]. Using this approaches leads to generation inhomogenous distribution of temperature in annealed materials. Just this inhomogeneity leads to decreasing dimensions of elements of integrated circuits and their discrete analogs. Another approach to decrease the above dimensions is doping required areas of epitaxial layers of heterostructures by dopant diffusion or ion implantation. However optimization of annealing of dopant and/or radiation defects is required in this case $[18,19]$. It is also attracted an interest radiation processing of doped materials. The processing leads to changing distribution of concentration of dopants [20]. The changing could also leads to decrease dimensions of elements of integrated circuits and their discrete ana$\operatorname{logs}[21-23]$. 


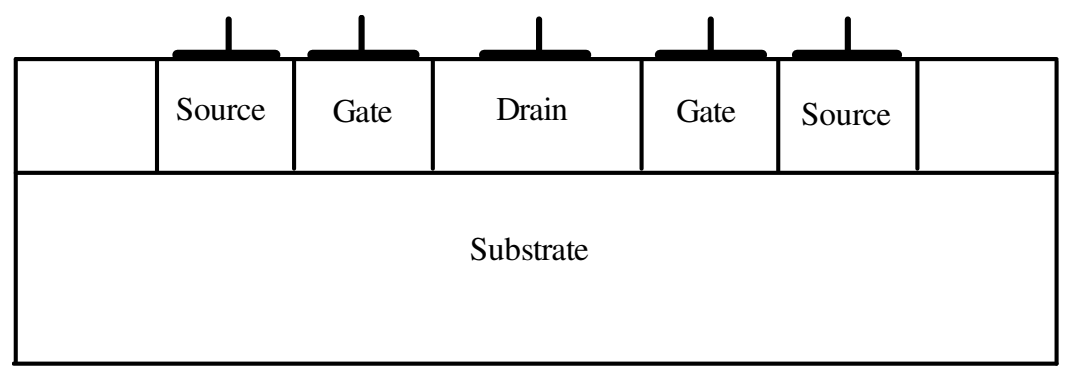

Fig. 1. Heterostructure, which consist of a substrate and epitaxial layer with several sections. Structure in deep of heterostructure

In this paper we analysed redistribution of concentration of dopant with account redistribution of radiation defects in the considered heterostructure, which is presented in Fig. 1. Some sections have been manufactured in epitaxial layer so, as it is shown in the Fig. 1. Dopants have been infused or implanted in the sections to produce required types of conductivity $(n$ or $p)$. Farther annealing of dopant and/or radiation defects should be annealed. Main aim of our paper is analysis of dynamic of redistribution of dopant and radiation defects in considered heterostructure during annealing.

\section{METHOD OF SOLUTION}

To solve our aims we determine spatio-temporal distribution of concentration of dopant. To determine the distribution one shall solve the following equation [1,3-5]

$$
\frac{\partial C(x, y, z, t)}{\partial t}=\frac{\partial}{\partial x}\left[D_{C} \frac{\partial C(x, y, z, t)}{\partial x}\right]+\frac{\partial}{\partial y}\left[D_{C} \frac{\partial C(x, y, z, t)}{\partial y}\right]+\frac{\partial}{\partial z}\left[D_{C} \frac{\partial C(x, y, z, t)}{\partial z}\right] .
$$

Boundary and initial conditions for the equation are

$$
\begin{gathered}
\left.\frac{\partial C(x, y, z, t)}{\partial x}\right|_{x=0}=0,\left.\frac{\partial C(x, y, z, t)}{\partial x}\right|_{x=L_{x}}=0,\left.\frac{\partial C(x, y, z, t)}{\partial y}\right|_{y=0}=0,\left.\frac{\partial C(x, y, z, t)}{\partial y}\right|_{x=L_{y}}=0, \\
\left.\frac{\partial C(x, y, z, t)}{\partial z}\right|_{z=0}=0,\left.\frac{\partial C(x, y, z, t)}{\partial z}\right|_{x=L_{z}}=0, C(x, y, z, 0)=f(x, y, z) .
\end{gathered}
$$

The function $C(x, y, z, t)$ describes the spatio-temporal distribution of concentration of dopant; $\mathrm{T}$ is the temperature of annealing; $\mathrm{D}_{\mathrm{C}}$ is the dopant diffusion coefficient. Dopant diffusion coefficient is different in different materials. The diffusion coefficient is also depends on temperature of annealing with account Arrhenius law. Dependences of dopant diffusion coefficients could be approximated by the following function [3,24-26]

$$
D_{C}=D_{L}(x, y, z, T)\left[1+\xi \frac{C^{\gamma}(x, y, z, t)}{P^{\gamma}(x, y, z, T)}\right]\left[1+\varsigma_{1} \frac{V(x, y, z, t)}{V^{*}}+\varsigma_{2} \frac{V^{2}(x, y, z, t)}{\left(V^{*}\right)^{2}}\right] .
$$

The multiplier $D_{L}(x, y, z, T)$ depends on coordinate and temperature (due to Arrhenius law); $P$ $(x, y, z, T)$ is the limit of solubility of dopant; the parameter $\gamma$ is different in different materials and should be integer in the following interval $\gamma \in[1,3][3]$; the function $V(x, y, z, t)$ describes the spa- 
tio-temporal distribution of concentration of radiation vacancies; parameter $V^{*}$ describes the equilibrium concentration of vacancies. Dependence of dopant diffusion coefficient on concentration has been described in details in [3]. It should be noted, that diffusive type of doping did not leads to radiation damage of materials and $\zeta_{1}=\zeta_{2}=0$. We determine spatio-temporal distributions of concentrations point radiation defects by solving the following system of equations $[25,26]$

$$
\left\{\begin{array}{l}
\frac{\partial I(x, y, z, t)}{\partial t}=\frac{\partial}{\partial x}\left[D_{I}(x, y, z, T) \frac{\partial I(x, y, z, t)}{\partial x}\right]+\frac{\partial}{\partial y}\left[D_{I}(x, y, z, T) \frac{\partial I(x, y, z, t)}{\partial y}\right]-I(x, y, z, t) \times \\
\times k_{I, V}(x, y, z, T) V(x, y, z, t)+\frac{\partial}{\partial z}\left[D_{I}(x, y, z, T) \frac{\partial I(x, y, z, t)}{\partial z}\right]-k_{I, I}(x, y, z, T) I^{2}(x, y, z, t) \\
\frac{\partial V(x, y, z, t)}{\partial t}=\frac{\partial}{\partial x}\left[D_{V}(x, y, z, T) \frac{\partial V(x, y, z, t)}{\partial x}\right]+\frac{\partial}{\partial y}\left[D_{V}(x, y, z, T) \frac{\partial V(x, y, z, t)}{\partial y}\right]-I(x, y, z, t) \times \\
\times k_{I, V}(x, y, z, T) V(x, y, z, t)+\frac{\partial}{\partial z}\left[D_{V}(x, y, z, T) \frac{\partial V(x, y, z, t)}{\partial z}\right]-k_{V, V}(x, y, z, T) V^{2}(x, y, z, t)
\end{array}\right.
$$

with boundary and boundary conditions

$$
\begin{gathered}
\left.\frac{\partial \rho(x, y, z, t)}{\partial x}\right|_{x=0}=0,\left.\frac{\partial \rho(x, y, z, t)}{\partial x}\right|_{x=L_{x}}=0,\left.\frac{\partial \rho(x, y, z, t)}{\partial y}\right|_{y=0}=0,\left.\frac{\partial \rho(x, y, z, t)}{\partial y}\right|_{y=L_{y}}=0, \\
\left.\frac{\partial \rho(x, y, z, t)}{\partial z}\right|_{z=0}=0,\left.\frac{\partial \rho(x, y, z, t)}{\partial z}\right|_{z=L_{z}}=0, \rho(x, y, z, 0)=f_{\rho}(x, y, z) .
\end{gathered}
$$

Here $\rho=I, V$; the function $I(x, y, z, t)$ describes the spatio-temporal distribution of concentration of interstitials; $D_{\rho}(x, y, z, T)$ are the diffusion coefficients of vacancies and interstitials; terms $V^{2}(x, y, z, t)$ and $I^{2}(x, y, z, t)$ corresponds to generation of divacancies and diinterstitials, respectively; $k_{I, V}(x, y, z, T), k_{I, I}(x, y, z, T)$ and $k_{V, V}(x, y, z, T)$ are the parameters of recombination of point radiation defects and generation their complexes, respectively.

Spatio-temporal distributions of concentrations of simplest complexes of radiation defects (divacancies $\Phi_{V}(x, y, z, t)$ and diinterstitials $\left.\Phi_{I}(x, y, z, t)\right)$ have been determine by solving by solving the following system of equations $[25,26]$

$$
\left\{\begin{array}{l}
\frac{\partial \Phi_{I}(x, y, z, t)}{\partial t}=\frac{\partial}{\partial x}\left[D_{\Phi I}(x, y, z, T) \frac{\partial \Phi_{I}(x, y, z, t)}{\partial x}\right]+\frac{\partial}{\partial y}\left[D_{\Phi I}(x, y, z, T) \frac{\partial \Phi_{I}(x, y, z, t)}{\partial y}\right]+ \\
+\frac{\partial}{\partial z}\left[D_{\Phi I}(x, y, z, T) \frac{\partial \Phi_{I}(x, y, z, t)}{\partial z}\right]+k_{I, I}(x, y, z, T) I^{2}(x, y, z, t)-k_{I}(x, y, z, T) I(x, y, z, t) \\
\frac{\partial \Phi_{V}(x, y, z, t)}{\partial t}=\frac{\partial}{\partial x}\left[D_{\Phi V}(x, y, z, T) \frac{\partial \Phi_{V}(x, y, z, t)}{\partial x}\right]+\frac{\partial}{\partial y}\left[D_{\Phi V}(x, y, z, T) \frac{\partial \Phi_{V}(x, y, z, t)}{\partial y}\right]+ \\
+\frac{\partial}{\partial z}\left[D_{\Phi V}(x, y, z, T) \frac{\partial \Phi_{V}(x, y, z, t)}{\partial z}\right]+k_{V, V}(x, y, z, T) V^{2}(x, y, z, t)-k_{V}(x, y, z, T) V(x, y, z, t)
\end{array}\right.
$$




$$
\left\{\begin{array}{l}
\frac{\partial \Phi_{I}(x, y, z, t)}{\partial t}=\frac{\partial}{\partial x}\left[D_{\Phi I}(x, y, z, T) \frac{\partial \Phi_{I}(x, y, z, t)}{\partial x}\right]+\frac{\partial}{\partial y}\left[D_{\Phi I}(x, y, z, T) \frac{\partial \Phi_{I}(x, y, z, t)}{\partial y}\right]+ \\
+\frac{\partial}{\partial z}\left[D_{\Phi I}(x, y, z, T) \frac{\partial \Phi_{I}(x, y, z, t)}{\partial z}\right]+k_{I, I}(x, y, z, T) I^{2}(x, y, z, t)-k_{I}(x, y, z, T) I(x, y, z, t) \\
\frac{\partial \Phi_{V}(x, y, z, t)}{\partial t}=\frac{\partial}{\partial x}\left[D_{\Phi V}(x, y, z, T) \frac{\partial \Phi_{V}(x, y, z, t)}{\partial x}\right]+\frac{\partial}{\partial y}\left[D_{\Phi V}(x, y, z, T) \frac{\partial \Phi_{V}(x, y, z, t)}{\partial y}\right]+ \\
+\frac{\partial}{\partial z}\left[D_{\Phi V}(x, y, z, T) \frac{\partial \Phi_{V}(x, y, z, t)}{\partial z}\right]+k_{V, V}(x, y, z, T) V^{2}(x, y, z, t)-k_{V}(x, y, z, T) V(x, y, z, t)
\end{array}\right.
$$

with boundary and initial conditions

$$
\begin{gathered}
\left.\frac{\partial \Phi_{\rho}(x, y, z, t)}{\partial x}\right|_{x=0}=0,\left.\frac{\partial \Phi_{\rho}(x, y, z, t)}{\partial x}\right|_{x=L_{x}}=0,\left.\frac{\partial \Phi_{\rho}(x, y, z, t)}{\partial y}\right|_{y=0}=0,\left.\frac{\partial \Phi_{\rho}(x, y, z, t)}{\partial y}\right|_{y=L_{y}}=0, \\
\left.\frac{\partial \Phi_{\rho}(x, y, z, t)}{\partial z}\right|_{z=0}=0,\left.\frac{\partial \Phi_{\rho}(x, y, z, t)}{\partial z}\right|_{z=L_{z}}=0, \Phi_{I}(x, y, z, 0)=f_{\phi I}(x, y, z), \Phi_{V}(x, y, z, 0)=f_{\Phi V}(x, y, z) .
\end{gathered}
$$

Here $D_{\Phi I}(x, y, z, T)$ and $D_{\Phi V}(x, y, z, T)$ are diffusion coefficients of diinterstitials and divacancies; $k_{I}$ $(x, y, z, T)$ and $k_{V}(x, y, z, T)$ are parameters of decay of complexes.

It should be noted, that nonlinear equations with space and time varying coefficients are usually used to describe physical processes. Although the equations are usually solved in different limiting cases [27-30]. Spatio-temporal distribution of concentration of dopant have been calculated by using method of averaging of function corrections [21,31] with decreased quantity of iteration steps [32]. Framework this approach we used solutions of the above differential equations without any nonlinearity and with averaged values of diffusion coefficients and thermal diffusivity $D_{0 L}$, $D_{0 I}, D_{0 V}, D_{0 \Phi I}, D_{0 \Phi V}, \alpha_{0}$. The solutions could be written as

$$
\begin{gathered}
C_{1}(x, y, z, t)=\frac{1}{L_{x} L_{y} L_{z}}+\frac{2}{L_{x} L_{y} L_{z}} \sum_{n=1}^{\infty} F_{n C} c_{n}(x) c_{n}(y) c_{n}(z) e_{n C}(t), \\
I_{1}(x, y, z, t)=\frac{1}{L_{x} L_{y} L_{z}}+\frac{2}{L_{x} L_{y} L_{z}} \sum_{n=1}^{\infty} F_{n I} c_{n}(x) c_{n}(y) c_{n}(z) e_{n I}(t), \\
V_{1}(x, y, z, t)=\frac{1}{L_{x} L_{y} L_{z}}+\frac{2}{L_{x} L_{y} L_{z}} \sum_{n=1}^{\infty} F_{n C} c_{n}(x) c_{n}(y) c_{n}(z) e_{n V}(t), \\
\Phi_{I 1}(x, y, z, t)=\frac{1}{L_{x} L_{y} L_{z}}+\frac{2}{L_{x} L_{y} L_{z}} \sum_{n=1}^{\infty} F_{n \Phi_{I}} c_{n}(x) c_{n}(y) c_{n}(z) e_{n \Phi_{I}}(t), \\
\Phi_{V 1}(x, y, z, t)=\frac{1}{L_{x} L_{y} L_{z}}+\frac{2}{L_{x} L_{y} L_{z}} \sum_{n=1}^{\infty} F_{n \Phi_{V}} c_{n}(x) c_{n}(y) c_{n}(z) e_{n \Phi_{V}}(t),
\end{gathered}
$$

where $e_{n \rho}(t)=\exp \left[-\pi^{2} n^{2} D_{0 \rho} t\left(\frac{1}{L_{x}^{2}}+\frac{1}{L_{y}^{2}}+\frac{1}{L_{z}^{2}}\right)\right], F_{n \rho}=\int_{0}^{L_{x}} c_{n}(u) \iint_{0}^{L_{y}} c_{n}(v) \int_{0}^{L_{z}} c_{n}(w) f_{\rho}(u, v, w) d w d v d u, c_{n}(\chi)$ $=\cos \left(\pi n \chi \mid L_{\chi}\right)$.

The second-order approximations and approximations with higher orders of concentration of dopant, concentrations of radiation defects and temperature have been calculated framework standard iteration procedure of method of averaging of function corrections [21,31,32]. Framework the approach to calculate $n$-th-order approximations of the above concentrations and temperature 
we replace the required functions $C(x, y, z, t), I(x, y, z, t), V(x, y, z, t), \Phi_{I}(x, y, z, t)$ and $\Phi_{V}(x, y, z, t)$ in the right sides of Eqs. (1), (4), (6) on the following sums $\alpha_{n}+\rho_{n-1}(x, y, z, t)$. The replacement gives us possibility to obtain the following equations for the second-order approximation of above concentrations

$$
\begin{aligned}
& \frac{\partial C_{2}(x, y, z, t)}{\partial t}=\frac{\partial}{\partial x}\left(D_{L}(x, y, z, T)\left[1+\varsigma_{1} \frac{V(x, y, z, t)}{V^{*}}+\varsigma_{2} \frac{V^{2}(x, y, z, t)}{\left(V^{*}\right)^{2}}\right]\left\{1+\xi \frac{\left[\alpha_{2 C}+C_{1}(x, y, z, t)\right]^{\gamma}}{P^{\gamma}(x, y, z, T)}\right\} \times\right. \\
& \left.\times \frac{\partial C_{1}(x, y, z, t)}{\partial x}\right)+\frac{\partial}{\partial y}\left(D_{L}(x, y, z, T)\left[1+\varsigma_{1} \frac{V(x, y, z, t)}{V^{*}}+\varsigma_{2} \frac{V^{2}(x, y, z, t)}{\left(V^{*}\right)^{2}}\right]\left\{1+\xi \frac{\left[\alpha_{2 C}+C_{1}(x, y, z, t)\right]^{\gamma}}{P^{\gamma}(x, y, z, T)}\right\} \frac{\partial C_{1}(x, y, z, t)}{\partial y}+(8)\right. \\
& +\frac{\partial}{\partial z}\left(D_{L}(x, y, z, T) \frac{\partial C_{1}(x, y, z, t)}{\partial z}\left\{1+\xi \frac{\left[\alpha_{2 C}+C_{1}(x, y, z, t)\right]^{\gamma}}{P^{\gamma}(x, y, z, T)}\right\}\left[1+\varsigma_{2} \frac{V^{2}(x, y, z, t)}{\left(V^{*}\right)^{2}}+\varsigma_{1} \frac{V(x, y, z, t)}{V^{*}}\right]\right) \\
& \int \frac{\partial I_{2}(x, y, z, t)}{\partial t}=\frac{\partial}{\partial x}\left[D_{I}(x, y, z, T) \frac{\partial I_{1}(x, y, z, t)}{\partial x}\right]+\frac{\partial}{\partial y}\left[D_{I}(x, y, z, T) \frac{\partial I_{1}(x, y, z, t)}{\partial y}\right]+ \\
& +\frac{\partial}{\partial z}\left[D_{I}(x, y, z, T) \frac{\partial I_{1}(x, y, z, t)}{\partial z}\right]-k_{I, V}(x, y, z, T)\left[\alpha_{2 I}+I_{1}(x, y, z, t)\right]\left[\alpha_{2 V}+V_{1}(x, y, z, t)\right]- \\
& -k_{I, I}(x, y, z, T)\left[\alpha_{2 I}+I_{1}(x, y, z, t)\right]^{2} \\
& \frac{\partial V_{2}(x, y, z, t)}{\partial t}=\frac{\partial}{\partial x}\left[D_{V}(x, y, z, T) \frac{\partial V_{1}(x, y, z, t)}{\partial x}\right]+\frac{\partial}{\partial y}\left[D_{V}(x, y, z, T) \frac{\partial V_{1}(x, y, z, t)}{\partial y}\right]+ \\
& +\frac{\partial}{\partial z}\left[D_{V}(x, y, z, T) \frac{\partial V_{1}(x, y, z, t)}{\partial z}\right]-k_{I, V}(x, y, z, T)\left[\alpha_{2 I}+I_{1}(x, y, z, t)\right]\left[\alpha_{2 V}+V_{1}(x, y, z, t)\right]- \\
& -k_{V, V}(x, y, z, T)\left[\alpha_{2 V}+V_{1}(x, y, z, t)\right]^{2} \\
& \int \frac{\partial \Phi_{I 2}(x, y, z, t)}{\partial t}=\frac{\partial}{\partial x}\left[D_{\Phi I}(x, y, z, T) \frac{\partial \Phi_{I 1}(x, y, z, t)}{\partial x}\right]+\frac{\partial}{\partial y}\left[D_{\Phi I}(x, y, z, T) \frac{\partial \Phi_{I 1}(x, y, z, t)}{\partial x}\right]+ \\
& +\frac{\partial}{\partial z}\left[D_{\Phi I}(x, y, z, T) \frac{\partial \Phi_{I 1}(x, y, z, t)}{\partial z}\right]+k_{I, I}(x, y, z, T) I^{2}(x, y, z, t)-k_{I}(x, y, z, T) I(x, y, z, t) \\
& \frac{\partial \Phi_{V 2}(x, y, z, t)}{\partial t}=\frac{\partial}{\partial x}\left[D_{\Phi V}(x, y, z, T) \frac{\partial \Phi_{V 1}(x, y, z, t)}{\partial x}\right]+\frac{\partial}{\partial y}\left[D_{\Phi V}(x, y, z, T) \frac{\partial \Phi_{V 1}(x, y, z, t)}{\partial x}\right]+ \\
& +\frac{\partial}{\partial z}\left[D_{\Phi V}(x, y, z, T) \frac{\partial \Phi_{V 1}(x, y, z, t)}{\partial z}\right]+k_{V, V}(x, y, z, T) V^{2}(x, y, z, t)-k_{V}(x, y, z, T) V(x, y, z, t)
\end{aligned}
$$

Farther we obtain the second-order approximations of concentrations of dopant and radiation defects by integration of the left and right sides of the Eqs. (8)-(10)

$$
\begin{gathered}
C_{2}(x, y, z, t)=\frac{\partial}{\partial x}\left(\int_{0}^{t} D_{L}(x, y, z, T)\left[1+\varsigma_{1} \frac{V(x, y, z, \tau)}{V^{*}} \varsigma_{2} \frac{V^{2}(x, y, z, \tau)}{\left(V^{*}\right)^{2}}\right]\left\{1+\xi \frac{\left[\alpha_{2 C}+C_{1}(x, y, z, \tau)\right]^{\gamma}}{P^{\gamma}(x, y, z, T)}\right\} \times\right. \\
\left.\times \frac{\partial C_{1}(x, y, z, \tau)}{\partial x}\right) d \tau+\frac{\partial}{\partial y}\left(\int_{0}^{t}\left[1+\varsigma_{1} \frac{V(x, y, z, \tau)}{V^{*}} \varsigma_{2} \frac{V^{2}(x, y, z, \tau)}{\left(V^{*}\right)^{2}}\right]\left\{1+\xi \frac{\left[\alpha_{2 C}+C_{1}(x, y, z, \tau)\right]^{\gamma}}{P^{\gamma}(x, y, z, T)}\right\} \times\right. \\
\left.\times D_{L}(x, y, z, T) \frac{\partial C_{1}(x, y, z, \tau)}{\partial y}\right)+\frac{\partial}{\partial z}\left(\int_{0}^{t}\left[1+\varsigma_{1} \frac{V(x, y, z, \tau)}{V^{*}} \varsigma_{2} \frac{V^{2}(x, y, z, \tau)}{\left(V^{*}\right)^{2}}\right] \frac{\partial C_{1}(x, y, z, \tau)}{\partial y} \times\right.
\end{gathered}
$$


International Journal of Recent advances in Physics (IJRAP) Vol.4, No.1, February 2015

$$
\begin{aligned}
& \times D_{L}(x, y, z, T)\left\{1+\xi \frac{\left[\alpha_{2 C}+C_{1}(x, y, z, \tau)\right]^{\gamma}}{P^{\gamma}(x, y, z, T)}\right\}+f_{C}(x, y, z) \\
& I_{2}(x, y, z, t)=\frac{\partial}{\partial x}\left[\int_{0}^{t} D_{I}(x, y, z, T) \frac{\partial I_{1}(x, y, z, \tau)}{\partial x} d \tau\right]+\frac{\partial}{\partial y}\left[\int_{0}^{t} D_{I}(x, y, z, T) \frac{\partial I_{1}(x, y, z, \tau)}{\partial y} d \tau\right]+ \\
& +\frac{\partial}{\partial z}\left[\int_{0}^{t} D_{I}(x, y, z, T) \frac{\partial I_{1}(x, y, z, \tau)}{\partial z} d \tau\right]-\int_{0}^{t} k_{I, I}(x, y, z, T)\left[\alpha_{2 I}+I_{1}(x, y, z, \tau)\right]^{2} d \tau+f_{I}(x, y, z)- \\
& -\int_{0}^{t} k_{I, V}(x, y, z, T)\left[\alpha_{2 I}+I_{1}(x, y, z, \tau)\right]\left[\alpha_{2 V}+V_{1}(x, y, z, \tau)\right] d \tau \\
& \left\{V_{2}(x, y, z, t)=\frac{\partial}{\partial x}\left[\int_{0}^{t} D_{V}(x, y, z, T) \frac{\partial V_{1}(x, y, z, \tau)}{\partial x} d \tau\right]+\frac{\partial}{\partial y}\left[\int_{0}^{t} D_{V}(x, y, z, T) \frac{\partial V_{1}(x, y, z, \tau)}{\partial y} d \tau\right]+\right. \\
& +\frac{\partial}{\partial z}\left[\int_{0}^{t} D_{V}(x, y, z, T) \frac{\partial V_{1}(x, y, z, \tau)}{\partial z} d \tau\right]-\int_{0}^{t} k_{V, V}(x, y, z, T)\left[\alpha_{2 V}+V_{1}(x, y, z, \tau)\right]^{2} d \tau+f_{V}(x, y, z)- \\
& -\int_{0}^{t} k_{I, V}(x, y, z, T)\left[\alpha_{2 I}+I_{1}(x, y, z, \tau)\right]\left[\alpha_{2 V}+V_{1}(x, y, z, \tau)\right] d \tau \\
& \Phi_{I 2}(x, y, z, t)=\frac{\partial}{\partial x}\left[\int_{0}^{t} D_{\Phi I}(x, y, z, T) \frac{\partial \Phi_{I 1}(x, y, z, \tau)}{\partial x} d \tau\right]+\frac{\partial}{\partial y}\left[\int_{0}^{t} D_{\Phi I}(x, y, z, T) \frac{\partial \Phi_{I 1}(x, y, z, \tau)}{\partial y} d \tau\right]+ \\
& +\frac{\partial}{\partial z}\left[\int_{0}^{t} D_{\Phi I}(x, y, z, T) \frac{\partial \Phi_{I 1}(x, y, z, \tau)}{\partial z} d \tau\right]+f_{\Phi I}(x, y, z)+\int_{0}^{t} k_{I, I}(x, y, z, T) I^{2}(x, y, z, \tau) d \tau- \\
& -\int_{0}^{t} k_{I}(x, y, z, T) I(x, y, z, \tau) d \tau \\
& \left\{\Phi_{V 2}(x, y, z, t)=\frac{\partial}{\partial x}\left[\int_{0}^{t} D_{\Phi V}(x, y, z, T) \frac{\partial \Phi_{V 1}(x, y, z, \tau)}{\partial x} d \tau\right]+\frac{\partial}{\partial y}\left[\int_{0}^{t} D_{\Phi V}(x, y, z, T) \frac{\partial \Phi_{V 1}(x, y, z, \tau)}{\partial y} d \tau\right]+\right. \\
& +\frac{\partial}{\partial z}\left[\int_{0}^{t} D_{\Phi V}(x, y, z, T) \frac{\partial \Phi_{V 1}(x, y, z, \tau)}{\partial z} d \tau\right]+f_{\Phi V}(x, y, z)+\int_{0}^{t} k_{V, V}(x, y, z, T) V^{2}(x, y, z, \tau) d \tau- \\
& -\int_{0}^{t} k_{V}(x, y, z, T) V(x, y, z, \tau) d \tau
\end{aligned}
$$

We determine average values of the second-order approximations of the required functions by using the following standard relations [21,31,32]

$$
\alpha_{2 \rho}=\frac{1}{\Theta L_{x} L_{y} L_{z}} \int_{0}^{\Theta} \int_{0}^{L_{x}} \int_{0}^{L_{y}} \int_{0}^{L_{z}}\left[\rho_{2}(x, y, z, t)-\rho_{1}(x, y, z, t)\right] d z d y d x d t .
$$

Relations for the average values $\alpha_{2 \rho}$ could be obtain by substitution of the second-order approximations of the considered concentrations $(8 a)-(10 a)$ and their the first-order approximations into the relation (11)

$$
\alpha_{2 C}=\frac{1}{L_{x} L_{y} L_{z}} \int_{0}^{L_{x}} \int_{0}^{L_{y}} \int_{0}^{L_{z}} f_{C}(x, y, z) d z d y d x
$$


International Journal of Recent advances in Physics (IJRAP) Vol.4, No.1, February 2015

$$
\left\{\begin{array}{l}
\alpha_{2 I}=\frac{1}{2 A_{I I 00}}\left\{\left(1+A_{I V 01}+A_{I I 10}+\alpha_{2 V} A_{I V 00}\right)^{2}-4 A_{I I 00}\left[\alpha_{2 V} A_{I V 10}-\frac{1}{L_{x} L_{y} L_{z}} \int_{0}^{L_{x}} \int_{0}^{L_{y}} \int_{0}^{L_{z}} f_{I}(x, y, z) d z d y d x-A_{I I 20}+\right.\right. \\
\left.\left.+A_{I V 11}\right]\right\}^{\frac{1}{2}}-\frac{1+A_{I V 01}+A_{I I 10}+\alpha_{2 V} A_{I V 00}}{2 A_{I I 00}} \\
\alpha_{2 V}=\frac{1}{2 B_{4}} \sqrt{\frac{\left(B_{3}+A\right)^{2}}{4}-4 B_{4}\left(y+\frac{B_{3} y-B_{1}}{A}\right)}-\frac{B_{3}+A}{4 B_{4}},
\end{array}\right.
$$

where $A_{a b i j}=\frac{1}{\Theta L_{x} L_{y} L_{z}} \int_{0}^{\Theta}(\Theta-t) \int_{0}^{L_{x}} \int_{0}^{L_{y}} \int_{0}^{L_{z}} k_{a, b}(x, y, z, T) I_{1}^{i}(x, y, z, t) V_{1}^{j}(x, y, z, t) d z d y d x d t, B_{4}=A_{I V 00}^{2} \times$ $\times A_{I V 00}^{2}-2\left(A_{I V 00}^{2}-A_{I I 00} A_{V V 00}\right)^{2}, B_{3}=A_{I V 00} A_{I V 00}^{2}+A_{I V 01} A_{I V 00}^{3}+A_{I V 00} A_{I I 10} A_{I V 00}^{2}+4\left(A_{I I 00} A_{V V 00}+\right.$ $\left.+A_{I V 00}^{2}\right)\left[2 A_{I V 01} A_{I V 00}+2 A_{I V 00}\left(1+A_{I V 01}+A_{I I 10}\right)-2 A_{I I 00}\left(A_{I V 10}+A_{V V 10}+1\right)\right]-4 A_{I V 10} A_{I I 00} A_{I V 00}^{2}+$ $+2 A_{I V 00} A_{I V 01} A_{I V 00}^{2}, B_{2}=\left\{A_{I V 00}^{2} A_{I V 01}^{2}-4\left[A_{I V 11}-A_{I I 20}-\frac{1}{L_{x} L_{y} L_{z}} \int_{0}^{L_{x}} \int_{0}^{L_{y}} \int_{0}^{L_{z}} f_{I}(x, y, z) d z d y d x\right] \times\right.$ $\left.\times 4 A_{I I 00}+\left(1+A_{I V 01}+A_{I I 10}\right)^{2}+2 A_{I V 00} A_{I V 01}\left(A_{I V 00} A_{I V 01}+A_{I V 00} A_{I I 10}-4 A_{I V 10} A_{I I 00}+A_{I V 00}\right)\right\} A_{I V 00}^{2}\left\{\left[2 A_{I V 01} A_{I V 00}+\right.\right.$ $\left.+2 A_{I V 00}\left(1+A_{I V 01}+A_{I I 10}\right)-2 A_{I I 00}\left(A_{I V 10}+A_{V V 10}+1\right)\right]^{2}+2\left[\frac{2}{L_{x} L_{y} L_{z}} \int_{0}^{L_{x}} \int_{0}^{L_{y}} \int_{0}^{L_{z}} f_{V}(x, y, z) d z d y d x+A_{I V 01}\left(A_{I I 10}+\right.\right.$ $\left.\left.+1+A_{I V 01}\right]-2 A_{I I 00}\left(A_{V V 20}-A_{I V 11}\right)+A_{I V 01}\left(1+A_{I V 01}+A_{I I 10}\right)\right]\left[2 A_{I V 00}\left(1+A_{I V 01}+A_{I I 10}\right)-2 A_{I I 00}\left(A_{I V 10}+A_{V V 10}+1\right)+\right.$ $\left.\left.+2 A_{I V 01} A_{I V 00}\right]\right\}, B_{1}=2 A_{I V 00} A_{I V 01}\left(1+A_{I V 01}+A_{I I 10}\right)^{2}-8\left[A_{I V 11}-\frac{1}{L_{x} L_{y} L_{z}} \int_{0}^{L_{x}} \int_{0}^{L_{y}} \int_{0}^{L_{z}} f_{I}(x, y, z) d z d y d x-\right.$ $\left.-A_{I I 20}\right] A_{I V 00} A_{I V 01} A_{I I 00}+A_{I V 01}^{2}\left(A_{I V 00}+A_{I V 00} A_{I V 01}+A_{I V 00} A_{I I 10}-4 A_{I V 10} A_{I I 00}\right)-2\left[\frac{2 A_{I I 00}}{L_{x} L_{y} L_{z}} \int_{0}^{L_{x}} \int_{0}^{L_{y}} \int_{0}^{L_{z}} f_{I}(x, y, z) d z d y d x+\right.$ $\left.+A_{I V 01}\left(1+A_{I V 01}+A_{I I 10}\right)-2 A_{I I 00}\left(A_{V V 20}-A_{I V 11}\right)+A_{I V 01}\left(1+A_{I V 01}+A_{I I 10}\right)\right]\left[2 A_{I V 00}\left(1+A_{I V 01}+A_{I I 10}\right)-\right.$ $\left.-2 A_{I I 00}\left(A_{I V 10}+A_{V V 10}+1\right)+2 A_{I V 01} A_{I V 00}\right], B_{0}=4 A_{I V 01}^{2} A_{I I 00}\left[\frac{1}{L_{x} L_{y} L_{z}} \int_{0}^{L_{x}} \int_{0}^{L_{y}} \int_{0}^{L_{z}} f_{I}(x, y, z) d z d y d x-\right.$ $\left.-A_{I V 11}+A_{I I 20}\right]+A_{I V 01}^{2}\left(A_{I V 01}+A_{I I 10}+1\right)^{2}-\left[\frac{2 A_{I I 00}}{L_{x} L_{y} L_{z}} \int_{0}^{L_{x}} \int_{0}^{L_{y}} \int_{0}^{L_{z}} f_{V}(x, y, z) d z d y d x+\left(1+A_{I V 01}+A_{I I 10}\right) \times\right.$ $\left.\times A_{I V 01}-2 A_{I I 00}\left(A_{V V 20}-A_{I V 11}\right)+A_{I V 01}\left(1+A_{I V 01}+A_{I I 10}\right)\right]^{2}, y=\sqrt[3]{\sqrt{q^{2}+p^{3}}-q}-\sqrt[3]{\sqrt{q^{2}+p^{3}}+q}+\frac{B_{2}}{6}$, $q=\frac{B_{2}^{3}}{216}+\frac{B_{0}\left(4 B_{2}-B_{3}^{2}\right)-B_{1}^{2}}{8}+\left(2 B_{1} B_{3}-8 B_{0}\right) \frac{B_{2}}{48}, p=\frac{B_{1} B_{3}}{12}-\frac{B_{0}}{3}-\frac{B_{2}^{2}}{36}, A=\sqrt{8 y+B_{3}^{2}-4 B_{2}}$, $\alpha_{2 \Phi_{I}}=A_{I I 20}-\frac{1}{\Theta L_{x} L_{y} L_{z}} \int_{0}^{\Theta}(\Theta-t) \int_{0}^{L_{x}} \int_{0}^{L_{y}} \int_{0}^{L_{z}} k_{I}(x, y, z, T) I(x, y, z, t) d z d y d x d t+$ $\left\{\begin{array}{r}+\frac{1}{L_{x} L_{y} L_{z}} \int_{0}^{L_{x}} \int_{0}^{L_{y}} \int_{0}^{L_{z}} f_{\Phi I}(x, y, z) d z d y d x \\ \alpha_{2 \Phi_{V}}=A_{V V 20}-\frac{1}{\Theta L_{x} L_{y} L_{z}} \int_{0}^{\Theta}(\Theta-t) \int_{0}^{L_{x}} \int_{0}^{L_{y}} \int_{0}^{L_{z}} k_{V}(x, y, z, T) V(x, y, z, t) d z d y d x d t \\ +\frac{1}{L_{x} L_{y} L_{z}} \int_{0}^{L_{x}} \int_{0}^{L_{y}} \int_{0}^{L_{z}} f_{\Phi V}(x, y, z) d z d y d x\end{array}\right.$

Value of the parameter $\alpha_{2 C}$ and final form of the appropriate equation depend on value of the parameter $\gamma$. 
Farther we analyzed spatio-temporal distributions of concentrations of dopant and radiation defects by using their the second-order approximations. Usually the second-order approximations of calculated values gives us possibility to obtain main physical results.

\section{DISCUSSION}

In this section we analyzed dynamics of redistribution of dopant and radiation defects during annealing. The Figs. 2 and 3 show distributions of concentrations of infused and implanted dopants in heterostructure, which consist of two layers, respectively. In this case we consider doping of sections of epitaxial layer in situation, when dopant diffusion coefficient in doped materials is larger, than in nearest areas. The Figs. 2 and 3 show, that presents of interface between materials of heterostructure gives us possibility to manufacture more compact field-effect transistor in comparison with field-effect transistor in homogenous materials.

To increase compactness the considered field-effect transistor it is attracted an interest optimization of annealing of dopant and/or radiation defects. Reason of this optimization is rather homogenous distribution of dopant and unnecessary doping of materials of heterostructure outside the considered sections. During short-time annealing dopant can not achieves interface between materials of heterostructure. We optimize annealing time framework recently introduced criterion $[18,19,21-23]$. Framework the criterion we approximate real distributions of concentrations of dopants by step-wise functions. We minimize the following mean-squared error to estimate optimal values of annealing time

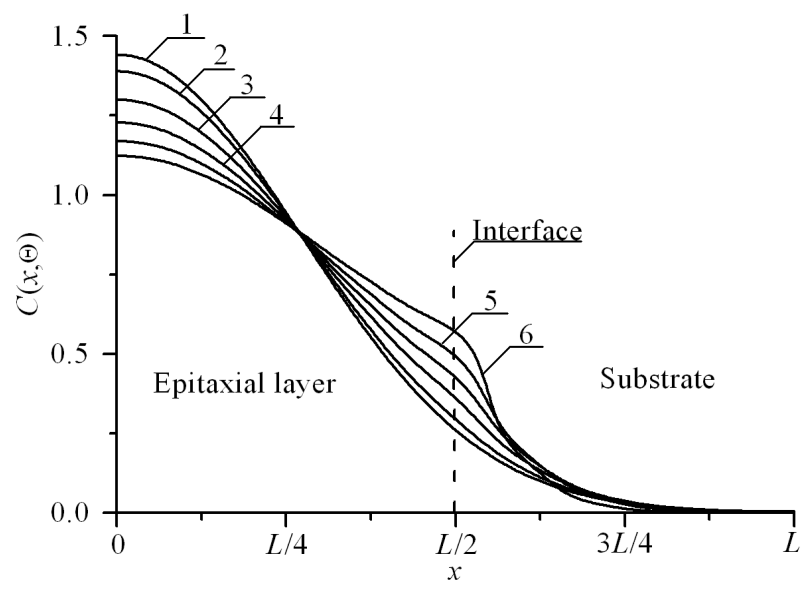

Fig.2. Typical distributions of concentration of dopant.

The dopant has been infused in the heterostructure from Fig. 1. The direction of the infusion is perpendicular to interface between epitaxial layer substrate. The distributions have been calculated under condition, when value of dopant diffusion coefficient in epitaxial layer is larger, than value of dopant diffusion coefficient in substrate. Increasing of number of curves corresponds to increasing of difference between values of dopant diffusion coefficient in layers of heterostructure 


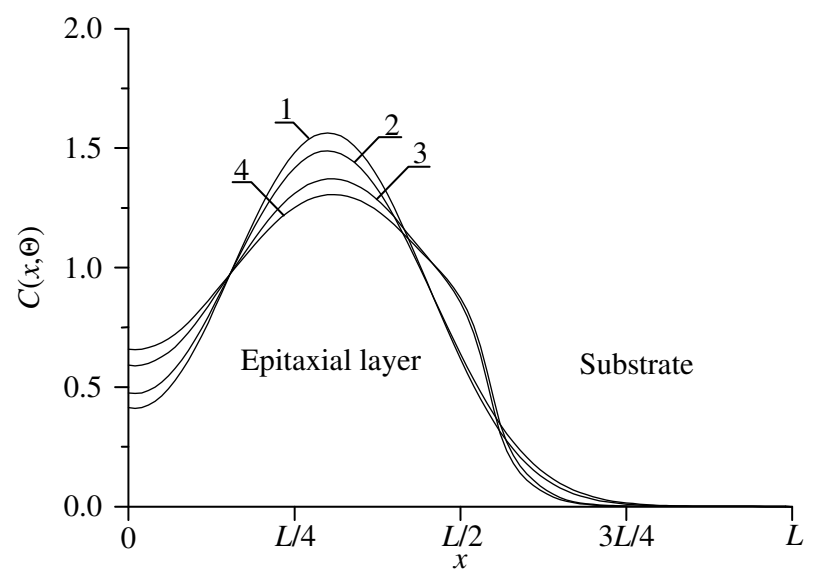

Fig.3. Typical distributions of concentration of dopant.

The dopant has been implanted in the heterostructure from Fig. 1. The direction of the implanted is perpendicular to interface between epitaxial layer substrate. The distributions have been calculated under condition, when value of dopant diffusion coefficient in epitaxial layer is larger, than value of dopant diffusion coefficient in substrate. Increasing of number of curves corresponds to increasing of difference between values of dopant diffusion coefficient in layers of heterostructure. Curves 1 and 3 corresponds to annealing time $\Theta=0.0048\left(L_{x}{ }^{2}+L_{y}{ }^{2}+L_{z}{ }^{2}\right) / D_{0}$. Curves 2 and 4 corresponds to annealing time $\Theta=0.0057\left(L_{x}{ }^{2}+L_{y}{ }^{2}+L_{z}{ }^{2}\right) / D_{0}$.

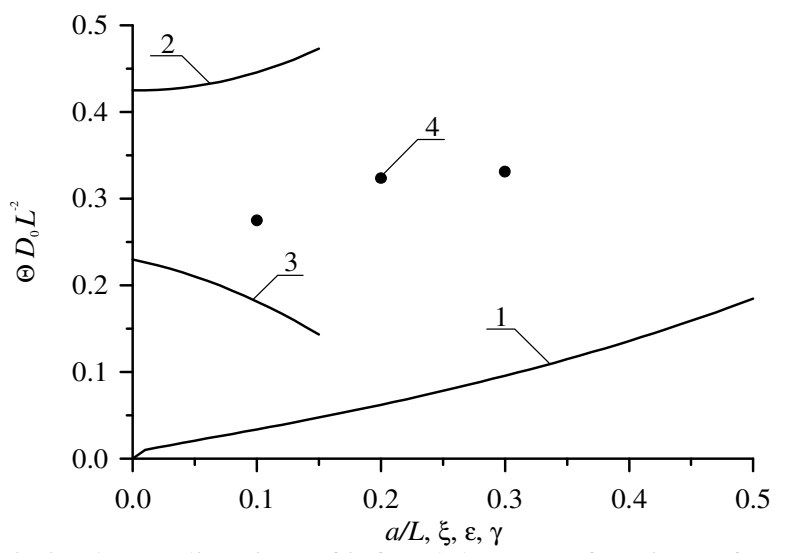

Fig.4. Optimized annealing time of infused dopant as functions of parameters.

Curve 1 describes dependence of annealing time on the relation $a / L$ for $\xi=\gamma=0$ for equal to each other values of dopant diffusion coefficient in all parts of heterostructure. Curve 2 describes dependence of annealing time on the parameter $\mathcal{E}$ for $a / L=1 / 2$ and $\xi=\gamma=0$. Curve 3 describes dependence of annealing time on the parameter $\xi$ for $a / L=1 / 2$ and $\xi=\gamma=0$. Curve 4 describes dependence of annealing time on the parameter $\gamma$ for $a / L=1 / 2$ and $\varepsilon=\xi=0$ 


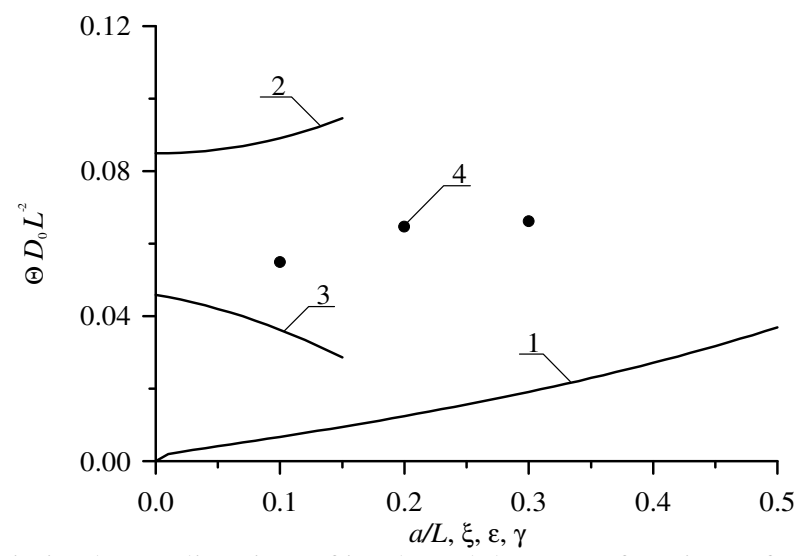

Fig.5. Optimized annealing time of implanted dopant as functions of parameters.

Curve 1 describes dependence of annealing time on the relation $a / L$ for $\xi=\gamma=0$ for equal to each other values of dopant diffusion coefficient in all parts of heterostructure. Curve 2 describes dependence of annealing time on the parameter $\varepsilon$ for $a / L=1 / 2$ and $\xi=\gamma=0$. Curve 3 describes dependence of annealing time on the parameter $\xi$ for $a / L=1 / 2$ and $\xi=\gamma=0$. Curve 4 describes dependence of annealing time on the parameter $\gamma$ for $a / L=1 / 2$ and $\varepsilon=\xi=0$

$$
U=\frac{1}{L_{x} L_{y} L_{z}} \int_{0}^{L_{x}} \int_{0}^{L_{y}} \int_{0}^{L_{z}}[C(x, y, z, \Theta)-\psi(x, y, z)] d z d y d x .
$$

Here $\psi(x, y, z)$ is the idealised step-wise distribution of concentration of dopant, which would like to obtain for maximal decreasing of dimensions of transistors. Dependences of optimal values of annealing time are presented on Figs. 4 and 5 for diffusion and ion types of doping, respectively. It should be noted, that after finishing implantation of ions of dopant it is necessary to anneal of radiation of defects. It could be find spreading of distribution of dopant during the annealing. In the ideal case distribution of dopant achieves interface between materials of heterostructure during annealing of radiation defects. It is necessary to anneal dopant after finishing of annealing of radiation defects in the case, when the dopant did not achieves the interface between layers of heterostructure during annealing of radiation defects. In this situation optimal value of continuance of additional annealing is smaller, than continuance of annealing of infused dopant. It should be noted, that introduced approach to increase integration rate of field-effect transistors gives us possibility to simplify their common construction.

\section{Conclusions}

In this paper we introduce an approach to increase integration rate of field-effect heterotransistors. Framework the approach one should manufacture a heterostructure with special construction. After that appropriate areas of the heterostructure with account construction should be doped by diffusion or ion implantation. After the doping dopant and/or radiation defects should be annealed. It has been formulated a recommendation to optimize annealing to

The approach based on manufacture heterostructure with special construction, doping of required areas of heterostructure by dopant diffusion or ion implantation and optimization of annealing of dopant and/or radiation defects. The optimization of annealing gives us possibility to decrease dimensions of transistors with increasing their integration rate. At the same time one can obtain simplification of construction of integrated circuits. 
International Journal of Recent advances in Physics (IJRAP) Vol.4, No.1, February 2015

\section{ACKNOWLEDGMENTS}

This work is supported by the contract 11.G34.31.0066 of the Russian Federation Government, grant of Scientific School of Russia, the agreement of August 27, 2013 № 02.B.49.21.0003 between The Ministry of education and science of the Russian Federation and Lobachevsky State University of Nizhni Novgorod and educational fellowship for scientific research of Nizhny Novgorod State University of Architecture and Civil Engineering.

\section{REFERENCES}

[1] I.P. Stepanenko. Basis of Microelectronics (Soviet Radio, Moscow, 1980).

[2] A.G. Alexenko, I.I. Shagurin. Microcircuitry. Moscow: Radio and communication, 1990.

[3] Z.Yu. Gotra, Technology of microelectronic devices (Radio and communication, Moscow, 1991).

[4] N.A. Avaev, Yu.E. Naumov, V.T. Frolkin. Basis of microelectronics (Radio and communication, Mos-cow, 1991).

[5] V.I. Lachin, N.S. Savelov. Electronics. Rostov-on-Don: Phoenix, 2001.

[6] A. Polishscuk. "Ultrashallow p+-n junctions in silicon: electron-beam diagnostics of sub-surface region.". Modern Electronics. Issue 12. P. 8-11 (2004).

[7] G. Volovich. "Integration of on-chip field-effect transistor switches with dopantless Si/SiGe quantum dots for high-throughput testing". Modern Electronics. Issue 2. P. 10-17 (2006).

[8] A. Kerentsev, V. Lanin. Design and technological features of MOSFETs, Power Electronics. Issue 1. P. 34 (2008).

[9] A.O. Ageev, A.E. Belyaev, N.S. Boltovets, V.N. Ivanov, R.V. Konakova, Ya.Ya. Kudrik, P.M. Litvin, V.V. Milenin, A.V. Sachenko. "Au-TiBx-n-6H-SiC Schottky barrier diodes: the features of current flow in rectifying and nonrectifying contacts". Semiconductors. Vol. 43 (7). P. 897-903 (2009).

[10] Jung-Hui Tsai, Shao-Yen Chiu, Wen-Shiung Lour, Der-Feng Guo. "Au-TiBx-n-6H-SiC Schottky barrier diodes: the features of current flow in rectifying and nonrectifying contacts.।". Semiconductors. Vol. 43 (7). P. 971-974 (2009).

[11] E.I. Gol'dman, N.F. Kukharskaya, V.G. Naryshkina, G.V. Chuchueva. "The manifestation of excessive centers of the electron-hole pair generation, appeared as a result to field and thermal stresses, and their subsequent annihilation in the dynamic current-voltage characteristics of Si-MOS-structures with the ultrathin oxide". Semiconductors. Vol. 45 (7). P. 974-979 (2011).

[12] T.Y. Peng, S.Y. Chen, L.C. Hsieh C.K. Lo, Y.W. Huang, W.C. Chien, Y.D. Yao. "Impedance behavior of spin-valve transistor". J. Appl. Phys. Vol. 99 (8). P. 08H710-08H712 (2006).

[13] W. Ou-Yang, M. Weis, D. Taguchi, X. Chen, T. Manaka, M. Iwamoto. "Modeling of threshold voltage in pentacene organic field-effect transistors". J. Appl. Phys. Vol. 107 (12). P. 124506-124510 (2010).

[14] J. Wang, L. Wang, L. Wang, Z. Hao, Yi Luo, A. Dempewolf, M. M ller, F. Bertram, J rgen Christen. "An improved carrier rate model to evaluate internal quantum efficiency and analyze efficiency droop origin of InGaN based light-emitting diodes". J. Appl. Phys. Vol. 112 (2). P. 023107-023112 (2012).

[15] K.K. Ong, K.L. Pey, P.S. Lee, A.T.S. Wee, X.C. Wang, Y.F. Chong. "Dopant distribution in the recrystallization transient at the maximum melt depth induced by laser annealing". Appl. Phys. Lett. 89 (17), 172111-172114 (2006).

[16] H.T. Wang, L.S. Tan, E. F. Chor. "Pulsed laser annealing of Be-implanted GaN". J. Appl. Phys. 98 (9), 094901-094905 (2006).

[17] Yu.V. Bykov, A.G. Yeremeev, N.A. Zharova, I.V. Plotnikov, K.I. Rybakov, M.N. Drozdov, Yu.N. Drozdov, V.D. Skupov. "Diffusion processes in semiconductor structures during microwave annealing". Radiophysics and Quantum Electronics. Vol. 43 (3). P. 836-843 (2003).

[18] E.L. Pankratov. "Dopant Diffusion dynamics and optimal diffusion time as influenced by diffusioncoefficient nonuniformity". Russian Microelectronics. 2007. V.36 (1). P. 33-39.

[19] E.L. Pankratov. "Redistribution of a dopant during annealing of radiation defects in a multilayer structure by laser scans for production of an implanted-junction rectifier". Int. J. Nanoscience. Vol. 7 (4-5). P. 187-197 (2008).

[20] V.V. Kozlivsky. Modification of semiconductors by proton beams (Nauka, Sant-Peterburg, 2003, in Russian). 
International Journal of Recent advances in Physics (IJRAP) Vol.4, No.1, February 2015

[21] E.L. Pankratov, E.A. Bulaeva. "Doping of materials during manufacture $\mathrm{p}-\mathrm{n}$-junctions and bipolar transistors. analytical approaches to model technological approaches and ways of optimization of distributions of dopants". Reviews in Theoretical Science. Vol. 1 (1). P. 58-82 (2013).

[22] E.L. Pankratov, E.A. Bulaeva. "Optimization of manufacture of field-effect heterotransistors without p-n-junctions to decrease their dimensions". International Journal of Recent Advances in Physics. Vol. 3 (3). P. 1-16 (2014).

[23] E.L. Pankratov, E.A. Bulaeva. "Analytical approach to model mass transport in a heterostructure during manufacturing an implanted-junction rectifiers". Quantum matter. Vol. 3 (5). P. 454-459 (2014).

[24] E.I. Zorin, P.V. Pavlov and D.I. Tetelbaum. Ion doping of semiconductors. Moscow: Energy, 1975.

[25] P.M. Fahey, P.B. Griffin, J.D. Plummer. "Point defects and dopant diffusion in silicon". Rev. Mod. Phys. 1989. V. 61. № 2. P. 289-388.

[26] V.L. Vinetskiy, G.A. Kholodar'. Radiative physics of semiconductors. ("Naukova Dumka", Kiev, 1979, in Russian).

[27] W.-X. Ni, G.V. Hansson, J.-E. Sundgren, L. Hultman, L.R. Wallenberg, J.-Y. Yao, L.C. Markert, J.E. Greene, Phys. Rev. B. Vol. 46 (12). P. $7551-7558$ (1992)

[28] B.A. Zon, S.B. Ledovsky, A.N. Likholet. Tech. Phys. Vol. 45 (4). P. 419-424 (2000).

[29] F. Faupel, W. Frank, M.-P. Macht, H. Mehrer, V. Naundorf, K. Ratzke, H. R. Schober, S.K. Sharma, H. Teichler. Reviews of modern physics. Vol. 75 (1). P. 237-280 (2003).

[30] S.A. Bahrani, Y. Jannot, A. Degiovanni. J. Appl. Phys. Vol. 114 (14). P. 143509-143516 (2014).

[31] Yu.D. Sokolov. "On the determination of the dynamic forces in mine hoist ropes". Applied Mechanics. Vol.1 (1). P. 23-35 (1955).

[32] E.L. Pankratov. "Dynamics of delta-dopant redistribution during heterostructure growth". The Europe-an Physical Journal B. 2007. V. 57, №3. P. 251-256.

\section{Authors:}

Pankratov Evgeny Leonidovich was born at 1977. From 1985 to 1995 he was educated in a secondary school in Nizhny Novgorod. From 1995 to 2004 he was educated in Nizhny Novgorod State University: from 1995 to 1999 it was bachelor course in Radiophysics, from 1999 to 2001 it was master course in Radiophysics with specialization in Statistical Radiophysics, from 2001 to 2004 it was PhD course in Radiophysics. From 2004 to 2008 E.L. Pankratov was a leading technologist in Institute for Physics of Microstructures. From 2008 to 2012 E.L. Pankratov was a senior lecture/Associate Professor of Nizhny Novgorod State University of Architecture and Civil Engineering. Now E.L. Pankratov is in his Full Doctor course in Radiophysical Department of Nizhny Novgorod State University. He has 110 published papers in area of his researches.

Bulaeva Elena Alexeevna was born at 1991. From 1997 to 2007 she was educated in secondary school of village Kochunovo of Nizhny Novgorod region. From 2007 to 2009 she was educated in boarding school "Center for gifted children". From 2009 she is a student of Nizhny Novgorod State University of Architecture and Civil Engineering (spatiality "Assessment and management of real estate"). At the same time she is a student of courses "Translator in the field of professional communication" and "Design (interior art)" in the University. E.A. Bulaeva was a contributor of grant of President of Russia (grant № MK-548.2010.2). She has 63 published papers in area of her researches. 